\title{
Sur quelques étrangers exotiques dans la prose contemporaine russe
}

\section{Anastasia Forquenot de La Fortelle}

\section{(2) OpenEdition}

1 Journals

Édition électronique

URL : http://journals.openedition.org/edl/438

DOI : $10.4000 /$ edl. 438

ISSN : 2296-5084

Éditeur

Université de Lausanne

\section{Édition imprimée}

Date de publication : 15 septembre 2009

Pagination : 253-262

ISBN : 978-2-940331-20-8

ISSN : 0014-2026

\section{Référence électronique}

Anastasia Forquenot de La Fortelle, "Sur quelques étrangers exotiques dans la prose contemporaine russe », Études de lettres [En ligne], 2-3 | 2009, mis en ligne le 15 septembre 2012, consulté le 22

décembre 2020. URL : http://journals.openedition.org/edl/438; DOI : https://doi.org/10.4000/edl.438 


\section{SUR QUELQUES ÉTRANGERS EXOTIQUES DANS LA PROSE CONTEMPORAINE RUSSE}

Au centre du présent article se trouve le roman de Denis Goutsko, Un Russophone, puisant son écriture dans le chaos babylonien social et spirituel de la Russie contemporaine et proposant à son lecteur une vision apocalyptique de la réalité postsoviétique. L'accueil d'une altérité exotique y rime avec «écueil» et l'auto-identification de l'individu s'y structure à travers la notion d'auto-étrangéité inconditionnelle.

La critique littéraire contemporaine a remarqué à plusieurs reprises qu'avec la désintégration de l'empire soviétique, la littérature russe s'est remplie de toutes sortes de personnages marginaux, handicapés tant sur le plan physique que spirituel. Des enfants irrévocablement rejetés par le monde des adultes, des adolescents ne trouvant point leur place dans la réalité qui les entoure, des rescapés d'une guerre qui a mutilé leur corps et leur âme, etc. - une pléiade de personnages «infirmes» qui inonde les pages de la prose de la fin du XX $\mathrm{XX}^{\mathrm{e}}$ et du début de XXI $\mathrm{X}^{\mathrm{e}}$ siècle.

Dans certains textes, ce vecteur de marginalité structurant un espace des "hommes de trop" (version moderne) rejoint celui de la dimension exotique. C'est le cas, par exemple, du roman d'Andreï Dmitriev La Baie de la joie ${ }^{1}$ où l'insolite ethnologique et culturel joue un rôle important. Dans ce texte, le héros est victime d'un complot de plusieurs escrocs ayant des vues sur son appartement. Afin de s'en emparer à un prix ridicule, ils attirent le héros dans une petite station balnéaire du nom de $\mathrm{La}$ Baie de la joie - un monde bien clos, marginalisé, se trouvant comme quelque part à la périphérie de l'existence et gouverné par ses propres

I. A. Dmitriev, Buhta radosti. 
lois et coutumes. Cet univers exotique rempli de petits restaurants arméniens, azerbaïdjanais, plongé dans les odeurs envoûtantes de la cuisine orientale ${ }^{2}$, le héros commence à le découvrir avec une certaine méfiance qui cède par la suite la place à la fascination. De ce point de vue, Dmitriev n'est point original: l'altérité, la différence exotique lui servent quelque part de support romantique et utopique afin de décrire un univers intègre, solide où les relations qui unissent les personnes entre elles sont celles de solidarité et sont dénuées d'une quelconque perversité comme celle qui gouverne, par exemple, l'univers des "chasseurs" d'appartements.

Par ailleurs, l'exotisme de tous ces Ichkhan, Artour et Karina - de ces «rois" des brochettes à l'orientale de la Baie de la joie, qui ont fui la violence sévissant sur leur terre natale et, implantés sur un sol étranger, ont formé une communauté d'où toute intolérance et haine nationale se trouvent chassées - est décrit chez Dmitriev à travers une tout autre perspective, trouvant ses origines dans cette dimension agressive de rejet d'un monde ethnique autre qui s'est activée d'une manière manifeste dans la réalité postsoviétique. Ainsi les habitants de la Baie de la joie sont-ils victimes de rackets réguliers organisés, entre autres, par la milice russe.

Cette dimension d'hostilité à toute manifestation d'étrangéité marquée par une forte couleur locale, ethnique et culturelle, atteint son acmé dans un autre texte contemporain qui crée un type de personnage bien particulier.

En 2005, le prix Booker est accordé au roman de Denis Goutsko Un Russophone. Ce texte à forte inspiration autobiographique se divise en deux parties, dont la première relate l'expérience du service militaire de Mitia Vakoula (le récit de cette expérience inclut des passages où le héros se souvient de son enfance), un personnage-double de l'auteur, par les principaux jalons de son parcours biographique: comme son créateur, Mitia est né à Tbilissi dans une famille russe, il part faire ses études supérieures à Rostov et se trouve très rapidement envoyé accomplir son "devoir de citoyen" dans les points chauds du pays vivant une désintégration violente et douloureuse. La deuxième partie est consacrée au retour à la "vie normale», non militaire, mais qui se révèle entièrement

2. Ce monde est décrit à travers un décor de convention. 
marquée et même conditionnée par les épreuves de l'expérience précédente.

Le statut du héros dans le texte de Goutsko est bien particulier. Mitia Vakoula est constamment perçu par son entourage comme un élément inhabituel, exotique, irrévocablement autre par rapport au contexte où il se retrouve:

Il y a quelque chose d'indéfini. Comme une aura. On ne peut ni la voir ni la toucher, mais tu comprends: un étranger [...] Mitia a toujours senti lui-même sa différence [...] par rapport aux autres. Comme si on l'avait rajouté sur un dessin [...] tout le tableau est peint au pastel, tandis que lui est dessiné au crayon ${ }^{3}$.

En Russie, ce Russe géorgien incarne par son comportement et son fonctionnement un certain ensemble de caractéristiques - la manière de parler, de s'habiller, de faire la cuisine, etc. - qui font de lui quelqu'un de différent et d'exotique. Mais, d'une manière paradoxale, cette différence exotique se fait ressentir de la même manière dans la réalité géorgienne, où la famille de Mitia et le héros lui-même continuent à fonctionner en partie «à la russe» (sa grand-mère, par exemple, parle un mélange de russe et de géorgien provoquant des réactions d'étonnement amusé de la part de son entourage).

Ainsi, au centre du texte de Goutsko, se trouve un personnage dont le statut serait le mieux déterminé par le terme d'étranger. Mais il s'agit d'un étranger peu habituel. En effet, d'après les définitions communément admises, la situation d'étranger suppose un état provisoire, l'étrangéité étant une condition transitoire. Comme l'écrivent Joachim Manzi et Frédérique Toudoire-Surlapierre: «L'étranger n’est pas une identité mais un point de vue actualisé, une caractérisation subjective [...] l'étranger est cet autre qui ne doit jamais être complètement assimilé à l'autre» ${ }^{4}$. Or, l'étrangéité et l'exotisme de Mitia ne correspondent pas à ces définitions, car ils ne se présentent pas comme une identification passagère, mais semblent structurer une dénomination identitaire définitive. Le texte de Goutsko crée un personnage qui se caractérise par une sorte d'exotisme inconditionnel: indépendamment

3. D. Gucko, Russkogovorjaščij, p. 236. 1117.

4. J. Manzi, F. Toudoire-Surlapierre, "L'Inconnu qui frappe à ma porte», p. 1116- 
du contexte dans lequel Mitia se retrouve, il est perçu de toutes façons comme un élément étrange et étranger.

Déjà le titre du roman codifie cette particularité du héros qui, constante tout au long du roman, constitue la principale «caractéristique» de son identité. Cette dernière est indiquée par une référence à la langue qui ne rattache pas le héros d'une manière certaine à un lieu de provenance et à un espace précis d'identification. D'ailleurs, ce sont justement les particularités de la langue que parle le héros qui, dans le contexte russe, contribuent en premier lieu à faire de Mitia un personnage exotique. L’empreinte définitive dont la Géorgie a marqué ce Russe se ressent dès le premier contact, à travers un accent oriental très fort: «Tu as un de ces accents!» 5 . De même en Géorgie, c'est également la langue qui, certes, cette fois-ci, d'une manière moins directe, exprime, entre autres, une hétérogénéité exotique du héros par rapport à son entourage. La perception de la réalité chez Mitia est celle d'une conscience qui n'a jamais de prise directe sur le réel, mais qui l'appréhende à travers une optique biaisée par la prédominance d'un "second plan ». Le fonctionnement de ce dernier est entièrement conditionné par cette particularité du héros qui est traduite chez Goutsko de la manière suivante: "Il y a plus de littérature en lui que d'érythrocytes " ${ }^{6}$. Mitia fonctionne dans la réalité géorgienne en l'abordant à travers le prisme de ses lectures de la littérature russe et en étant perçu à nouveau comme un élément irrévocablement différent et étonnant par les gens qui se trouvent alentour. D'ailleurs, c'est également en commençant par la langue que le héros de Goutsko essaie de combattre son étrangéité et son exotisme inconditionnels en adoptant ce qu'il appelle une "pratique assidue du mimétisme " ${ }^{7}$. Car dès les premières pages du roman apparaît la dimension qui en fin de compte se révèle prédominante dans la relation unissant le héros au monde extérieur. Au-delà d'une curiosité étonnée l'étrangéité de Mitia suscite chez autrui une méfiance, se transformant rapidement en hostilité.

Ainsi, dans un dialogue entre Mitia et un interlocuteur anonyme, placé au tout début du roman, ce dernier, dans un mouvement manifeste de rejet, tente de faire accepter au héros - tout à fait gratuitement

5. D. Gucko, Russkogovorjaščij, p. 7-8.

6. Ibid., p.13.

7. Ibid., p. 11. 
- qu'il vaut mieux porter le prénom Dima. Par la suite, Mitia se retrouve dans la ville azerbaïdjanaise de Sheki, où il fait son service militaire et où, à travers le contexte de la guerre provoquée par la haine nationale, il accède à la connaissance authentique de sa propre identité: il réalise pleinement son statut particulier d'étranger inconditionnel qui provoque (à la différence de l'époque tbilissienne où l'exotisme du héros suscitait une simple curiosité) toutes sortes de manifestations violentes de rejet de la part de son entourage. Dans l'armée, Mitia commence à souffrir de l'ambiguïté de ses origines, sa situation existentielle se révèle proche de celle de ces Arméniens qui ont toujours vécu en Azerbaïdjan, dont certains ne parlent même plus l'arménien et ne sont plus chrétiens, mais qui se trouvent dans le pire des cas tués, dans le meilleur - chassés de l'Azerbaïdjan à cause de leurs origines. Par ailleurs, ces Arméniens se font persécuter, voire tuer - paradoxe tragique - non seulement par leurs voisins azeris, mais également par les Arméniens qui sont censés les accueillir sur le sol de leur patrie historique, mais qui ne veulent pas d'eux, les traitant comme des traittres convertis à l'Islam et ayant honteusement oublié leur langue d'origine. De ce point de vue, un des épisodes très forts du roman est celui qui décrit le bus avec les Arméniens fuyant Sheki (sous le contrôle de l'armée russe) et se trouvant de manière inattendue sous le feu de soldats arméniens.

Comme eux, Mitia se sent un marginal qui ne semble pouvoir ni s'enraciner ni se stabiliser dans un lieu fixe. Comme dans le cas de ces Arméniens qu'il est censé défendre, son statut de marginal «inconditionnel» le place dans un rapport particulier avec le monde extérieur, dans une relation qui pourrait être définie comme celle de l'espoir d'être accueilli quelque part, de l'espoir, selon l'expression de Jacques Derrida, d'une "hospitalité inconditionnelle». Mais le besoin de cette dernière, comme le démontre Derrida, peut recevoir en réponse une haine tout aussi inconditionnelle, car plus l'attente de la générosité est grande, plus le potentiel de haine et de rejet augmente lui aussi. Derrida cite à ce propos Hannah Arendt qui constate qu'avant la Seconde Guerre mondiale, l'on "assistait déjà à des déplacements massifs non d'exilés, mais de populations sans statut et sans garantie étatique...». Ces errances nomades constituaient "une sorte d'appel à l'hospitalité pure» ${ }^{8}$ et

8. J. Derrida, «Une Hospitalité à l’infini », p. 100. 
provoquaient un déchaînement de haine et d'hostilité beaucoup plus fort que lors de «formes d'exil classiques» 9 .

En accomplissant son service militaire, Mitia devient une des cibles privilégiées de cette violence inconditionnelle, "cruelle et irascible comme un tireur d'élite aux yeux de taupe " ${ }^{10}$. L'univers de l'armée ne représente en fin de compte qu'un micro-modèle de ce qui se passe à l'extérieur, sur le territoire azerbaïdjanais. Comme à l'extérieur, où la violence sévit contre ceux qui sont les plus proches, mais dérangent par leur différence (les voisins arméniens), les lois fondamentales qui conditionnent les relations entre les militaires et qui se trouvent encore renforcées par les dangers de la guerre sont celles - et là nous utilisons une fois de plus le terme que choisit Derrida en 1997 comme titre d'un de ses séminaires - "d'hostipitalité». Derrida l'emploie pour démontrer comment devant la crainte de la différence, les relations entre les êtres humains ne sont plus celles qui réunissent les deux hôtes bienveillants: le mot hôte «réalise» sa charge étymologique originelle, il s' «hostilise» et l'hospitalité se métamorphose en son contraire, en "hostipitalité ». La plus «vitale» parmi ces lois d'hostipitalité que découvre Mitia dans l'armée se formule de la manière suivante: «[...] le plus important, c'est de savoir qui précisément il ne faut pas aimer ${ }^{11}$. Mitia, que la guerre met à l'épreuve par rapport à son statut d'étranger inconditionnel, se trouve comme dans un cercle existentiel vicieux, formé de tensions contradictoires qui se nient mutuellement sans jamais se libérer dans une troisième entité autre: il est violemment haï par ses "camarades" de guerre aussi bien à cause de ses attaches géorgiennes qu'à cause de ses origines russes.

Cette haine est à l'origine de plusieurs incidents dans lesquels le héros est menacé d'être tué et dont le côté dangereux vaut celui des opérations militaires proprement dites. Malgré tous ses efforts pour s'adapter et pour résister (le héros essaie, afin de ne pas être rejeté, sinon d'être comme les autres, tout au moins de parler comme son entourage: une des stratégies mimétiques qu'il applique consiste à essayer de cacher devant les Russes son accent géorgien), Mitia ne trouve pas de réponse à

9. Voir à ce sujet, notre article «Un Russophone de Denis Goutsko: hospitalité ou "hostipitalité"?".

Io. D. Gucko, Russkogovorjaščij, p. 11.

II. Ibid., p. 9. 
cette question cruciale - «Mais, mon Dieu, dis-moi, souffle-moi qui je suis!» ${ }^{12}$ - et commence à ressentir son existence comme dénuée de tout sens. Dans le cercle vicieux dont il est prisonnier, le héros n'arrive plus à comprendre où se trouvent ses vraies attaches, sa vraie vie. Il ressent que son seul point de repère ontologique est un "point zéro", celui d'un vide, d'un gouffre béant (les images d'espace vide, de trous, de lacunes, d'abîmes sont récurrentes dans le texte):

[...] Mitia a ressenti que derrière son dos il y a bien maintenant un précipice, un énorme zéro insondable ${ }^{13}$.

Cette auto-représentation existentielle sera celle du héros tout au long du roman et lui sera sans cesse "confirmée» par l'attitude du monde qui l'entoure. Ainsi, par exemple, la notion de maison semble renvoyer, dans le cas de Mitia, à un vide, à une absence, à ce même "point zéro". Dans l'armée, il rêve de son «chez soi » à Tbilissi, mais cette image s'abolit lorsqu'elle se confronte à la réalité. En revenant dans la capitale géorgienne marquée par le déchaînement des conflits nationaux, le héros s'y sent étranger et se met à penser à Rostov. Mais quand il arrive dans la ville russe, ce même mécanisme de rejet du réel se remet en place. Cette absence de vraie maison est confirmée par les événements extérieurs: après la dislocation de l'URSS, Mitia est rejeté socialement de partout; il devient un citoyen de nulle part, car en Géorgie, il n'est pas accepté à cause de ses origines russes, et en Russie on ne lui délivre pas de passeport à cause de sa naissance en Géorgie.

Par ailleurs, cette connaissance de son statut d'étranger inconditionnel, d'«homme de nulle part» rejeté par tout le monde et dont l'existence est dénuée de tout sens, est indissolublement liée, dans son cas, à une autre découverte traumatisante: les mécanismes qui régissent le fonctionnement de l'univers postsoviétique, les rapports des gens entre eux et leur attitude envers eux-mêmes sont ceux d'un chaos absolu:

Tout est fragile, sans forme, tout coule, tout se déchire. Et en premier lieu les gens. Ils n'ont pas de point d'ancrage en eux... Ils sont liquides,

I2. Ibid., p. 143.

I3. Ibid., p. 58. 
fluides, ils coulent d'une forme vers une autre. Ils se liquéfient quand on y touche, fuient entre les doigts... C'est le chaos qui les modèle ${ }^{14}$.

Et c'est ainsi que l'image du personnage principal du texte de Denis Goutsko semble se doter d'une puissance métaphorique: elle traduit les particularités de la relation qui unit l'altérité à l'ipséité, ainsi que celles qui caractérisent l'auto-identification de l'homme dans le contexte du chaos babylonien du monde postsoviétique. La réalité de ce dernier est celle où les gens, livrés à eux-mêmes, ne savent plus gérer ni leur propre identité ni la différence, où ils souffrent d'un sentiment permanent d'auto-étrangéité et où chaque manifestation de divergence exotique est condamnée d'emblée à recevoir un accueil hostile.

Anastasia Forquenot de La Fortelle

Lausanne

I4. Ibid., p. 136. 


\section{BIBLIOGRAPHIE}

Bertrandias, B., "Hostilité d'une inquiétante étrangeté», in Le Livre de l'hospitalité: accueil de l'étranger dans l'histoire et les cultures, A. Montandon (dir.), Paris, Bayard, 2004.

Derrida, J., "Une Hospitalité à l'infini », in Manifeste pour l'hospitalité, Autour de Jacques Derrida, Grigny, Editions Paroles d'Aubes, 1999.

Dmitriev, A., Buxta radosti, Moskva, Vremja, 2008.

Gucko, D., Ruskogovorjaščij, Moskva, Vagrius, 2006.

Manzi, J., Toudoire-Surlapierre, F., "L'Inconnu qui frappe à ma porte", in Le Livre de l'hospitalité: accueil de l'étranger dans l'histoire et les cultures, A. Montandon (dir.), Paris, Bayard, 2004.

Ricceur, P., Soi-même comme un autre, Paris, Seuil, 1990.

Vinogradova-de La Fortelle, A., "Un Russophone de Denis Goutsko: hospitalité ou "hostipitalité"?», in L'Hôte étranger dans la culture russe, Caen, Presses de l'Université de Caen, à paraître. 
